\title{
Antiglutamate Receptor Antibodies and Cognitive Impairment in Primary Antiphospholipid Syndrome and Systemic Lupus Erythematosus
}

\begin{abstract}
Maria Gerosa ${ }^{1,2}$, Barbara Poletti ${ }^{3}$, Francesca Pregnolato ${ }^{4}$, Gabriella Castellino ${ }^{5}$, Annalisa Lafronza $^{3}$, Vincenzo Silani ${ }^{3,6}$, Piersandro Riboldi ${ }^{1,5}$, Pier Luigi Meroni ${ }^{1,4 *}$ and Joan T. Merrill ${ }^{7}$

${ }^{1}$ Department of Clinical Sciences and Community Health, University of Milan, Milan, Italy, ${ }^{2}$ Division of Rheumatology, Lupus Clinic, Istituto Ortopedico Gaetano Pini, Milan, Italy, ${ }^{3}$ Department of Neurology-Stroke Unit and Laboratory of Neuroscience, IRCCS Istituto Auxologico Italiano, Milan, Italy, ${ }^{4}$ Experimental Laboratory of Immunological and Rheumatologic Researches, IRCCS Istituto Auxologico Italiano, Milan, Italy, ${ }^{5}$ Allergy, Clinical Immunology and Rheumatology Unit, IRCCS Istituto Auxologico Italiano, Milan, Italy, ${ }^{6}$ Department of Pathophysiology and Transplantation, "Dino Ferrari" Center, Università degli Studi di Milano, Milan, Italy, ${ }^{7}$ Clinical Pharmacology Research Program, Oklahoma Medical Research Foundation, University of Oklahoma, Oklahoma City, OK, USA
\end{abstract}

Systemic lupus erythematosus (SLE) and antiphospholipid syndrome have an increased risk to develop cognitive impairment. A possible role for antiphospholipid antibodies (aPL) and antiglutamate receptor (anti-NMDA) antibodies in the pathogenesis of neurological manifestations of these two conditions, have been suggested. In particular, the role of anti-NMDA antibodies in the pathogenesis of neuropsychiatric SLE is supported by several experimental studies in animal models and by the finding of a correlation between anti-NMDA positivity in cerebrospinal fluid and neurological manifestations of SLE. However, data from the literature are controversial, as several studies have reported a correlation of these antibodies with mild cognitive impairment in SLE, but more recent studies have not confirmed this finding. The synergism between anti-NMDA and other concomitant autoantibodies, such as aPL, can be hypothesized to play a role in inducing the tissue damage and eventually the functional abnormalities. In line with this hypothesis, we have found a high incidence of at least one impaired cognitive domain in a small cohort of patients with primary APS (PAPS) and SLE. Interestingly, aPL were associated with low scoring for language ability and attention while anti-NMDA titers and mini-mental state examination scoring were inversely correlated. However, when patients were stratified according to the presence/absence of aPL, the correlation was confirmed in aPL positive patients only. Should those findings be confirmed, the etiology of the prevalent defects found in PAPS patients as well as the synergism between aPL and anti-NMDA antibodies would need to be explored.

Keywords: systemic lupus erythematosus, antiphospholipid syndrome, mild cognitive impairment, neuropsychological assessment, central nervous system involvement, anti-NMDA/glutamate receptor antibodies

Abbreviations: ANA, antinuclear antibodies; anti-CL, anticardiolipin; anti-dsDNA, antidouble strand DNA; anti-PL, antiphospholipid antibodies; anti- $\beta 2 \mathrm{GPI}$, anti- $\beta 2$ glycoprotein I; APS, antiphospholipid syndrome; BBB, blood-brain barrier; CCI, cognitive impairment index; CNS, central nervous system; CSF, cerebrospinal fluid; ELISA, enzyme-linked immunosorbent assay; ENA, extractable nuclear antigen; ICV, intracerebroventricular; Ig, immunoglobulins; LA, lupus anticoagulant; MMSE, mini-mental state examination; NMDA, human glutamate receptor; NPSLE, neuropsychiatric SLE; PAPS, primary APS; SLE, systemic lupus erythematosus. 


\section{INTRODUCTION}

Systemic lupus erythematosus (SLE) is a chronic disease characterized by antibodies directed to different components of the cell nucleus in association with a variety of clinical manifestations, including skin rash, arthritis, serositis, nephritis, hematological cytopenias, and neurological manifestations. The prevalence ranges from 15 to 50 cases per 100,000 persons and the incidence is around 2-8 new cases per 100,000 persons per year. Polyclonal B-cell stimulation and autoantibody production, leading to immune complex deposition and complement activation represent the most important pathogenic mechanisms of the disease (1). Treatment of SLE largely depends on the type of clinical manifestations: antimalarial agents in association with low-dose steroids represent the first choice in mild disease, while immunosuppressant agents, such as azathioprine, methotrexate, cyclophosphamide, and mycophenolate mofetil are used in more severe manifestations. Recently, belimumab, a new biological agent able to modulate B lymphocyte function, has been demonstrated to reduce lupus disease activity (1).

Antiphospholipid syndrome (APS) is a systemic autoimmune disease close to SLE with significant overlap of serological and clinical characteristics (2). It is mainly characterized by pregnancy complications and thrombotic events, involving both the venous and the arterial district (2). The formal classification of APS requires the persistent presence of medium to high titers of antiphospholipid antibodies (anti-PL), namely anti- $\beta 2$ glycoprotein I (anti- $\beta 2 \mathrm{GPI}$ ), anticardiolipin (anti-CL), and lupus anticoagulant (LA) (2). The disease can occur as an isolate clinical entity [primary APS (PAPS)] or associated with other autoimmune diseases, mainly with SLE (secondary APS). The prevalence is estimated around $40-50$ cases per 100,000 persons and the incidence is around five new cases per 100,000 persons per year. Elevated levels of anti-PL and positivity to more than one test have been linked to a higher risk for developing the disease (3). Treatment of APS is based on prevention of recurrence and is mainly represented by long term anticoagulation. Hydroxychloroquine, statins, rituximab, and eculizumab can be considered in refractory cases (3).

\section{COGNITIVE IMPAIRMENT IN APS AND SLE}

Among the several neurological symptoms related to SLE and classified by the American College of Rheumatology (3), cognitive dysfunctions have been reported to affect $6-66 \%$ of the patients according to different studies, but the prevalence may be up to $95 \%$ when cognitive defects are assessed by computerized neuropsychological testing (4). This literature is confusing, and the real incidence of dementia or mild cognitive defects in SLE patients has not yet been well defined.

Neurological involvement is very frequent in APS, cerebral stroke being one of the most common vascular manifestations. In fact, ischemic events are the most frequently observed central nervous system (CNS) complications of PAPS and represent one of the formal classification criteria. Multi-infarct dementia is also described in these patients, mainly associated with recurrent ischemic events, with an incidence estimated as $10-56 \%$ increasing with age $(3,5,6)$. Additional manifestations, such as migraine, seizures, chorea, transverse myelopathy, and multiple sclerosis-like syndrome have been reported (3). Cognitive impairment has frequently been addressed as a neurological APS "non-classification criterion." However, this finding has usually been described in APS associated with an underlying systemic autoimmune disease in the majority of the reports, mainly in SLE-associated APS, while only two studies are available in the literature in PAPS $(5,6)$. A case-control study of 60 patients with primary or secondary APS found that $42 \%$ presented mild cognitive defects, primarily involving verbal fluency and attention. Looking at PAPS patients only, the prevalence was about the same (38\%); however, besides anti-PL, no further correlation with serological profiles was evaluated (5). Similar results have recently been reported in a study comparing the cognitive function of anti-PL positive non-SLE subjects and anti-PL negative SLE patients (6). In this study, 20 SLE anti-PL negative patients and 20 anti-PL positive subjects, with or without clinical manifestations of APS, were investigated through a standardized cognitive test battery; $60 \%$ of SLE and $40 \%$ of anti-PL positive subjects displayed a cognitive impairment assessed by a global cognitive impairment index (CCI). This finding was not associated to disease activity or duration, while a possible correlation with the whole serological profile was not assessed (6).

Cognitive defects in PAPS cannot be simply explained by ischemic events; therefore, a direct effect of anti-PL on neuronal cells was suggested. Accordingly, anti-PL were found to bind CNS cells in vitro (7) and the intrathecal passive transfer of class G immunoglobulins (IgG) from APS patients were shown to induce cognitive defects in mice in vivo (7). These results have been recently confirmed by the same group, which demonstrated that the intracerebroventricular (ICV) passive transfer of human anti-PL IgG purified from the serum of APS patients with CNS involvement induced specific hyperactivity in injected mice (7). In addition to anti-PL, PAPS patients can display several organ non-specific autoantibodies, such as ANA, anti-dsDNA, antinucleosome, and anti-extractable nuclear antigen (anti-ENA), even in the absence of evident clinical manifestations of SLE (3).

Lupus-specific mechanisms underlying neuropsychiatric disease are better known and have been related to vasculitides of intracranial vessels, local or systemic production of inflammatory mediators, and generation of specific autoantibodies (8). Different types of autoantibodies have been investigated, such as aPL themselves and antiribosomal P protein antibodies (4, 8, 9). An increasing interest has been raised during the last decade for antibodies reacting to the human glutamate receptor (antiNMDA) as potentially involved in the pathogenesis of neurological manifestations of SLE.

\section{ANTI-NMDA ANTIBODIES}

A murine monoclonal anti-DNA antibody has been recently shown to cross-react with the extracellular domain of mouse and human glutamate receptor (NMDA) and to induce cognitive dysfunction in mice through neuronal cell apoptosis (10). 
Affinity-purified anti-NMDA antibodies were demonstrated to induce apoptotic cell death in vitro, when added to neuronal cultures. Moreover, mice immunized with a specific antigen to produce anti-NMDA antibodies developed cognitive dysfunction and spatial memory impairment only when a blood-brain barrier (BBB) disruption occurred (10). The autoantibodies selectively bound to hippocampal neurons and caused neuronal death. Interestingly, the administration of memantine, an NMDA receptor antagonist, prior to $\mathrm{BBB}$ disruption, prevented neuronal damage (10). Moreover, the direct injection of antibodies eluted from the brain of a SLE patient with severe CNS involvement in the C57BL/6 mice hippocampus was able to induce neuronal loss (10). More recently, the same group has demonstrated that antiNMDA antibodies development was associated with selective impairment of spatial memory in female $\mathrm{BALB} / \mathrm{c}$ mice and that relevant structural abnormalities could be observed in the surviving hippocampal pyramidal neurons (11). Further investigations have shown the presence of similar anti-NMDA antibodies in both serum and cerebrospinal fluid (CSF) of SLE patients even if a clear clinical association between these autoantibodies and cognitive defects has not yet been established (12).
Anti-NMDA antibodies have been reported in SLE patients with a prevalence of $19-33 \%$ in different studies $(4,9)$. Given their high pathogenicity of these in experimental animal models, a possible relationship with neuropsychiatric manifestations of SLE was investigated. Some authors have reported an association between anti-NMDA and several neurological manifestations, such as cognitive impairment, decline in memory functions, impaired attention or executive functions, and depression. However, some other studies in very large cohorts of lupus patients have not confirmed these data $(4,9)$. Remarkably, an association between the presence of anti-NMDA in the CSF and NP-SLE (neuropsychiatric SLE) manifestations was demonstrated in different studies. Moreover, CSF anti-NMDA titers correlated with clinical manifestations and with evidence of BBB dysfunction (11). Data examining the PAPS population for anti-NMDA are scant. No correlation between the presence of anti-NMDA and anti-CL in a SLE population was previously found, but these autoantibodies have not been evaluated in selected cohorts of primary APS patients (4).

With this as a background, we have recently examined the incidence of cognitive dysfunctions in a cohort of consecutive
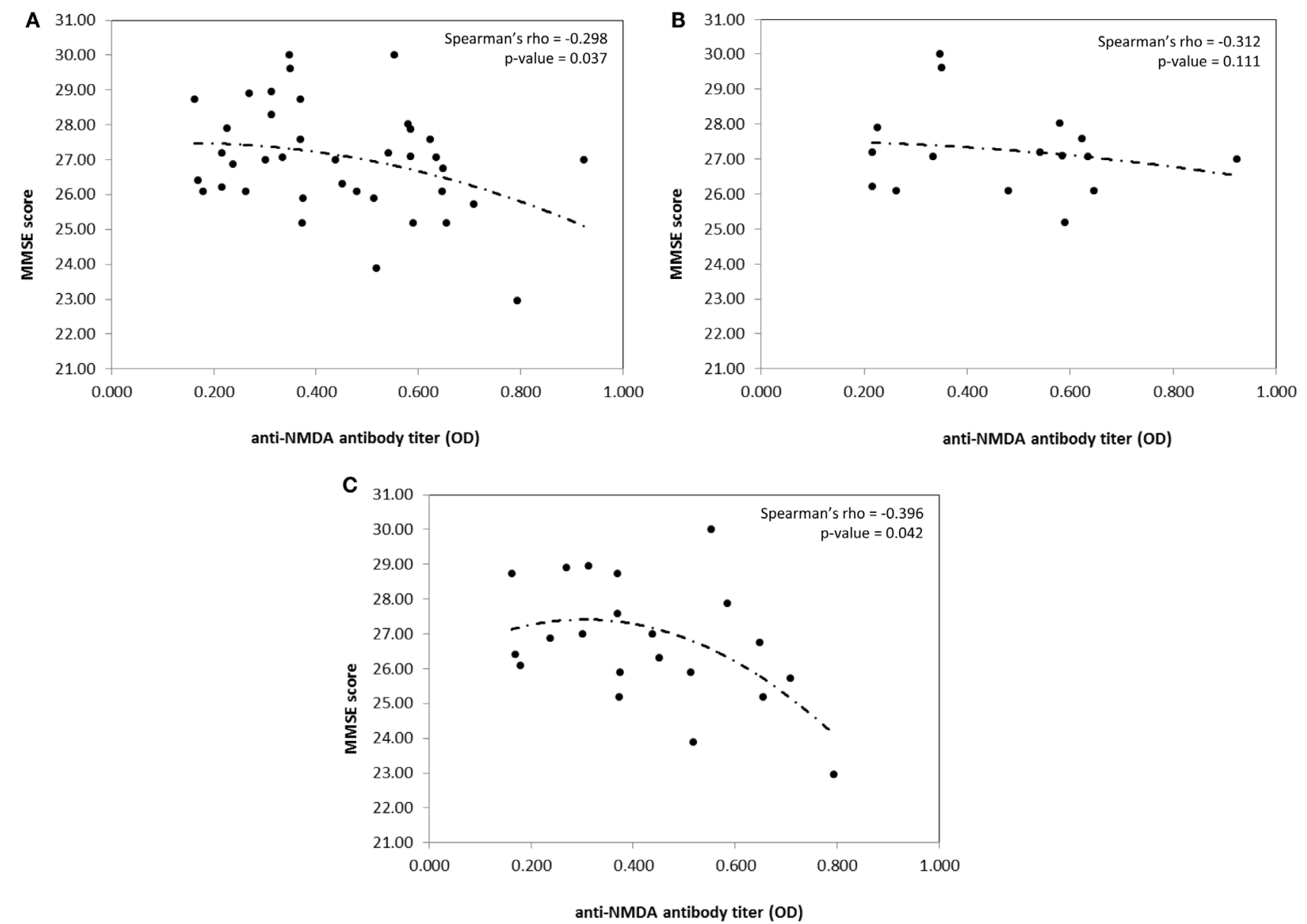

FIGURE 1 | Correlation between MMSE score and anti-NMDA antibody titer in the whole population (A), aPL negative patients (B), and aPL positive patients (C), only. Spearman's rho coefficient and relative $p$-value are reported on the top right corner of the graph. Curves are fitted according to a second polynomial equation model. 


\begin{tabular}{|c|c|c|c|}
\hline & & & Reference \\
\hline $\begin{array}{l}\text { In vitro } \\
\text { experiments }\end{array}$ & \multicolumn{2}{|c|}{$\begin{array}{l}\text { - Anti-NMDA ab from SLE pts cross-react with anti-dsDNA } \\
\text { - Affinity-purified anti-NMDA ab added to neuronal cultures induce apoptotic cell death } \\
\text { - Anti-NMDA/dsDNA cross-reactive ab induce "excitotoxicity" and neuronal apoptosis }\end{array}$} & $\begin{array}{l}\text { DeGiorgio et al. (13), Kowal } \\
\text { et al. (14), Kowal et al. (10), } \\
\text { Chang et al. (11) }\end{array}$ \\
\hline Humans & \multicolumn{2}{|c|}{ 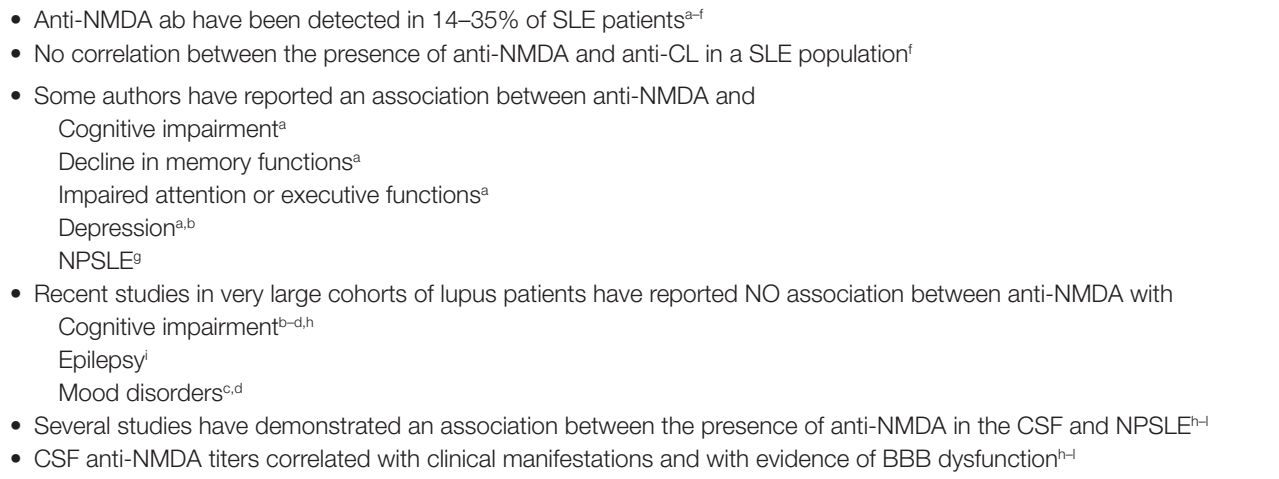 } & $\begin{array}{l}\text { a. Omdal et al. (15) } \\
\text { b. Lapteva et al. (16) } \\
\text { c. Harrison et al. (17) } \\
\text { d. Hanly et al. (9) } \\
\text { e. Kozora et al. (18) } \\
\text { f. Husebye et al. (19) } \\
\text { g. Gono et al. (20) } \\
\text { h. Hanly et al. (21) } \\
\text { i. Arinuma et al. (22) } \\
\text { j. Yoshio et al. (23) } \\
\text { k. Hirohata et al. (12) } \\
\text { l. Chang et al. (11) }\end{array}$ \\
\hline Our results & \multicolumn{2}{|c|}{$\begin{array}{l}\text { - } 27 \text { SLE and } 15 \text { PAPS underwent an extensive neuropsychological text battery } \\
\text { - } 44 \% \text { SLE and 33\% PAPS anti-NMDA ab positive (no differences in prevalence and titer) } \\
\text { - } 70 \% \text { SLE and } 86 \% \text { PAPS exhibited at least } 1 \text { defective domain } \\
\text { - } 11 \% \text { SLE and } 33 \% \text { PAPS exhibited } 4 \text { or more pathologic tests } \\
\text { - Mathematical abilities, attentional skills, and visual-motor planning were the most frequently affected cognitive domains } \\
\text { - PAPS patients significantly deviate from SLE patients in the Phonemic Verbal fluency and Street's Completion Test } \\
\text { - Anti-PL correlate with lower scoring for language ability and attention } \\
\text { - High anti-NMDA IgG titers correlate with low scoring for MMSE in the whole population and in anti-PL-positive } \\
\text { patients but NOT in aPL-negative patients independently from diagnosis }\end{array}$} & \\
\hline \multirow[t]{3}{*}{$\begin{array}{l}\text { Animal } \\
\text { models }\end{array}$} & $\begin{array}{l}\text { - } \text { BALB/c mice immunization to produce anti-NMDA antibodies or } \\
\text { - Intravenous infusion of serum from SLE pts, containing anti-NMDA ab in } \\
\text { BALB/c mice } \\
\text { - } \text { BBB abrogation induced by LPS }\end{array}$ & $\begin{array}{l}\text { Cognitive dysfunction and spatial } \\
\text { memory impairment development } \\
\text { Neuronal binding and apoptotic death } \\
\text { in hippocampus } \\
\text { Prevention of antibody-mediated injury } \\
\text { by the NMDA receptor antagonist } \\
\text { memantine }\end{array}$ & $\begin{array}{l}\text { DeGiorgio et al. (13), } \\
\text { Kowal et al. (14) }\end{array}$ \\
\hline & $\begin{array}{l}\text { - BALB/c mice immunization to produce anti-NMDA antibodies }{ }^{n} \\
\text { - BBB abrogation induced by epinephrine }{ }^{\circ}\end{array}$ & $\begin{array}{l}\text { Neuronal binding and apoptotic death } \\
\text { in amygdala } \\
\text { Aberrant pavlonian fear conditioning }\end{array}$ & $\begin{array}{l}\text { n. Kowal et al. (14) } \\
\text { o. Huerta et al. (24) }\end{array}$ \\
\hline & $\begin{array}{l}\text { - Direct injection of ab eluted from the brain of a severe NPSLE patient in the } \\
\text { C57BL/6 mice hippocampus }\end{array}$ & ab-mediated neuronal damage & Kowal et al. (10) \\
\hline
\end{tabular}

Ab, antibodies; pts, patients; BBB, blood-brain barrier; LPS, lipopolysaccharide; NPSLE, neuropsychiatric SLE; CSF, cerebrospinal fluid; MMSE, mini-mental state examination.

unselected PAPS in order to compare the results with those found in a parallel series of SLE patients and to better characterize the pattern of cognitive involvement in these two diseases and the possible associations between cognitive defects and the autoantibody profile. The protocol was approved by the Institutional Review Board (IRB) and informed consent was obtained from all the participants. Forty-two patients, 15 with a diagnosis of PAPS and 27 with a diagnosis of SLE, regularly followed in our Unit, underwent a 180 min neuropsychological test battery. Anti-NMDA levels were detected by a home-made enzyme-linked immunosorbent assay (ELISA) using a branched peptide as antigen (Asp Trp Glu Tyr Ser Val Trp Leu Ser Asn8 Lys4 Lys2 Lys- $\beta$ Ala) (9). Among SLE patients, all were ANA positive, three were positive for antiribosomal $\mathrm{P}$ antibodies, 13 for anti-dsDNA antibodies at medium/high titer, eight for LA, three for anti-CL IgG, five for anti- $\beta_{2}$ GPI IgG, two for anti-CL $\operatorname{IgM}$, and three for anti- $\beta_{2}$ GPI IgM at medium/high titer. Among
PAPS patients, ANA were positive in 8 and 2 were positive for medium/high titer anti-dsDNA antibodies. All patients had high titer anti-CL IgG and 14 patients high titer anti- $\beta_{2}$ GPI IgG, while LA was positive in 13 patients. Anti-NMDA antibodies were positive in 12 SLE and 5 PAPS, with no differences in prevalence and titer range. Considering the whole population, the incidence of cognitive dysfunctions was high since $76 \%$ of the population (13 PAPS and 19 SLE) exhibited at least one defective domain. In five PAPS and three SLE patients, four or more tests were pathologic and mathematical abilities, attentional skills, and visual-motor planning were the most frequently affected cognitive domains. However, when comparing score severity, PAPS patients significantly deviate from SLE patients in the Phonemic Verbal fluency $(U=121.5 ; p=0.018)$ and Street's Completion Test $(U=128.0 ; p=0.043)$. The presence of anti-PL was found to correlate with lower scoring for language ability and attention. A correlation between high anti-NMDA IgG titers and low scoring 
for the mini-mental state examination (MMSE) was found in the whole population (Rho $-0.298 ; p=0.037$ ). However, when patients were stratified according to the presence or the absence of anti-PL, the correlation was maintained in anti-PL positive patients only (Rho $-0.396 ; p=0.042$ ) (Figure 1). Higher antiNMDA titers were associated with low scores for the MMSE test, independently from the disease diagnosis. In Table $\mathbf{1}$ are summarized the results of the most important studies regarding anti-NMDA in SLE.

\section{COMMENTS}

Systemic lupus erythematosus patients are known to be more likely to develop cognitive impairment compared to the general population, and an association with anti-PL or anti-NMDA antibodies has been suggested $(4,5)$. Data from the literature are controversial, as several studies have reported a correlation of these antibodies with mild cognitive impairment in SLE, but more recent studies have not confirmed this finding. The role of anti-NMDA antibodies in the pathogenesis of NP-SLE is supported by experimental studies and by the finding of a correlation between anti-NMDA positivity in CSF and neurological manifestations of SLE. Not all the anti-NMDA positive patients display a BBB interruption that can allow circulating antibodies to reach the CNS and induce damage. Moreover, patients with previous BBB damage could recover, limiting access to the brain for the autoantibodies, which might continue to be measured in the peripheral blood. In this regard, the synergism between anti-NMDA and other concomitant autoantibodies could play a role in inducing the tissue damage and eventually the functional abnormalities. In line with this hypothesis, we can speculate that one of the potential mechanisms leading to BBB disruption could be represented by the endothelial perturbation induced by $\beta 2 \mathrm{GPI}$-dependent aPL, which could possibly facilitate the access of anti-NMDA antibodies in the cerebral circulation. Nevertheless, infections, CNS inflammation, or

\section{REFERENCES}

1. Lisnevskaia L, Murphy G, Isenberg D. Systemic lupus erythematosus. Lancet (2014) 384:1878-88. doi:10.1016/S0140-6736(14)60128-8

2. Miyakis S, Lockshin MD, Atsumi T, Branch DW, Brey RL, Cervera R, et al. International consensus statement on an update of the classification criteria for definite antiphospholipid syndrome (APS). J Thromb Haemost (2006) 4:295-306. doi:10.1111/j.1538-7836.2006.01753.x

3. Meroni PL, Chighizola CB, Rovelli F, Gerosa M. Antiphospholipid syndrome in 2014: more clinical manifestations, novel pathogenic players and emerging biomarkers. Arthritis Res Ther (2014) 16:209. doi:10.1186/ar4549

4. Hanly JG, Urowitz MB, Su L, Bae SC, Gordon C, Clarke A, et al. Autoantibodies as biomarkers for prediction of neuropsychiatric events in systemic lupus erythematosus. Ann Rheum Dis (2011) 70:1726-32. doi:10.1136/ard.2010.148502

5. Tektonidou MG, Varsou N, Kotoulas G, Antoniou A, Moutsopoulos HM. Cognitive deficits in patients with antiphospholipid sindrome. Arch Intern Med (2006) 166:2278-84. doi:10.1001/archinte.166.20.2278

6. Kozora E, Erkan D, Zhang L, Zimmerman R, Ramon G, Ulug AM, et al. Cognitive dysfunction in antiphospholipid antibody (aPL)-negative systemic lupus erythematosus (SLE) versus aPL-positive non-SLE patients. Clin Exp Rheumatol (2014) 32:34-40.

7. Katzav A, Ben-Ziv T, Blank M, Pick CG, Shoenfeld Y, Chapman J. Antibody-specific behavioral effects: intracerebroventricular injection other autoantibodies interaction can alternatively be implicated in BBB damage.

Our preliminary results are in accordance with this assumption. The occurrence of anti-NMDA antibodies in PAPS patients is not surprising. Anti-NMDA activity was originally described in anti-DNA antibodies derived from SLE mice (10) and antibodies to nuclear antigens can be found in PAPS (3).

In conclusion, both anti-PL and anti-NMDA antibodies behave as promising potential biomarkers of CNS involvement even if further longitudinal studies are essential to define the risk for a potential evolution and to also clarify the opportunity of starting a possible preventive therapy. Due to the great impact of cognitive impairment on quality of life and given the cognitive decline which has been described even in patients with a recent diagnosis, we propose that SLE and PAPS patients should be monitored for the development of cognitive impairment.

\section{AUTHOR CONTRIBUTIONS}

MG, BP, PR, and PM gave a substantial contribution to the conception and design of the work and interpretation of data. FP, AL, GC, VS, and JM gave a substantial contribution to acquisition, analysis, and interpretation of data. MG, BP, and PM participate in drafting the work. FP, GC, AL, PR, VS, and JM revised it critically for important intellectual content. MG, BP, FP, AL, GC, VS, $\mathrm{PR}, \mathrm{PM}$, and JM gave their final approval of the version to be published; and agreed to be accountable for all aspects of the work in ensuring that questions related to the accuracy or integrity of any part of the work are appropriately investigated and resolved.

\section{FUNDING}

The work was supported by the Gruppo LES Italiano - Gruppo Italiano per la Lotta contro il Lupus Eritematoso Sistemico - ONLUS (Premio per la ricerca e la cura del Lupus Eritematoso Sistemico).

of antiphospholipid antibodies induces hyperactive behavior while antiribosomal-P antibodies induces depression and smell deficits in mice. J Neuroimmunol (2014) 204:10-5. doi:10.1016/j.jneuroim.2014.04.003

8. Jennekens FG, Kater L. The central nervous system in systemic lupus erythematosus. Part 2. Pathogenetic mechanisms of clinical syndromes: a literature investigation. Rheumatology (2002) 41:619-30. doi:10.1093/ rheumatology/41.6.619

9. Hanly JG, Urowitz MB, Siannis F, Farewell V, Gordon C, Bae SC, et al. Autoantibodies and neuropsychiatric events at the time of systemic lupus erythematosus diagnosis. Results from an international inception cohort study. Arthritis Rheum (2008) 58:843-53. doi:10.1002/art.23218

10. Kowal C, DeGiorgio LA, Lee JY, Edgar MA, Huerta PT, Volpe BT, et al. Human lupus autoantibodies against NMDA receptors mediate cognitive impariment. Proc Natl Acad Sci U S A (2006) 103:1954-9. doi:10.1073/ pnas.0608397104

11. Chang EH, Volpe BT, Mackay M, Aranow C, Watson P, Kowal K, et al. Selective impairment of spatial cognition caused by autoantibodies to the N-methyl-D-aspartate receptor. EBioMedicine (2015) 2:755-64. doi:10.1016/j. ebiom.2015.05.027

12. Hirohata S, Arinuma Y, Yanagida T, Yoshio T. Blood-brain barrier damages and intrathecal synthesis of anti-N-methyl-D-aspartate receptor NR2 antibodies in diffuse psychiatric/neuropsychological syndromes in systemic lupus erythematosus. Arthritis Res Ther (2014) 16:R77. doi:10.1186/ar4518 
13. DeGiorgio LA, Konstantinov KN, Lee SC, Hardin JA, Volpe BT, Diamond B. A subset of lupus anti-DNA antibodies cross-reacts with the NR2 glutamate receptor in systemic lupus erythematosus. Nat Med (2001) 7:1189-93. doi:10.1038/nm1101-1189

14. Kowal C, DeGiorgio LA, Nakaoka T, Hetherington H, Huerta PT, Diamond B, et al. Cognition and immunity; antibody impairs memory. Immunity (2004) 21:179-88. doi:10.1016/j.immuni.2004.07.011

15. Omdal R, Brokstad K, Waterloo K, Koldingsnes W, Jonsson R, Mellegren SI. Neuropsychiatric disturbances in SLE are associated with antibodies against NMDA receptors. Eur J Neurol (2005) 12:392-8. doi:10.1111/j.1468-1331.2004.00976.x

16. Lapteva L, Nowak M, Yarboro CH, Takada K, Roebuck-Spencer T, Weickert T, et al. Anti-N-Metyl-D-Aspartate receptor antibodies, cognitive dysfunction, and depression in systemic lupus erythematosus. Arthtritis Rheum (2006) 54:2505-14. doi:10.1002/art.22031

17. Harrison MJ, Ravdin LD, Lockshin MD. Relationship between serum NR2a antibodies and cognitive dysfunction in systemic lupus erythematosus. Arthritis Rheum (2006) 54:2515-22. doi:10.1002/art.22030

18. Kozora E, Sterling GW, Maier SF, Filley CM, Arciniegas DB, Brown M, et al. Antibodies against $\mathrm{N}$-methyl-D-aspartate receptors in patients with systemic lupus erythematosus without major neuropsychiatric syndromes. J Neurol Sci (2010) 295:87-91. doi:10.1016/j.jns.2010.04.016

19. Husebye ES, Sthoeger ZM, Dayan M, Zinger H, Elbirt D, Levite M, et al. Autoantibodies to a NR2A peptide of the glutamate/NMDA receptor in sera of patients with systemic lupus erythematosus. Ann Rheum Dis (2005) 64:1210-3. doi:10.1136/ard.2004.029280

20. Gono T, Kawaguchi Y, Kaneko H, Nishimura K, Hanaoka M, Kataoka S, et al. Anti-NR2A antibody as a predictor for neuropsychiatric systemic lupus erythematosus. Rheumatology (2011) 50:1578-85. doi:10.1093/rheumatology/ keq408

21. Hanly JG, Su L, Omisade A, Farewell VT, Fisk JD. Screening for cognitive impairment in systemic lupus erythematosus. J Rheumatol (2012) 39:1371-7. doi:10.3899/jrheum.111504

22. Arinuma Y, Yanagida T, Hirohata S. Association of cerebrospinal fluid anti-NR2 glutamate receptor antibodies with diffuse neuropsychiatric systemic lupus erythematosus. Arthritis Rheum (2008) 58:1130-5. doi:10.1002/ art.23399

23. Yoshio T, Okamoto H, Hirohata S, Minota S. IgG anti-NR2 glutamate receptor autoantibodies from patients with systemic lupus erythematosus activate endothelial cells. Arthritis Rheum (2013) 65:457-63. doi:10.1002/ art. 37745

24. Huerta PT, Kowal C, DeGiorgio LA, Volpe BT, Diamond B. Immunity and behavior: antibodies alter emotion. Proc Natl Acad Sci (2006) 103:678-83. doi:10.1073/pnas.0510055103

Conflict of Interest Statement: The authors declare that the research was conducted in the absence of any commercial or financial relationships that could be construed as a potential conflict of interest.

Copyright (C) 2016 Gerosa, Poletti, Pregnolato, Castellino, Lafronza, Silani, Riboldi, Meroni and Merrill. This is an open-access article distributed under the terms of the Creative Commons Attribution License (CC BY). The use, distribution or reproduction in other forums is permitted, provided the original author(s) or licensor are credited and that the original publication in this journal is cited, in accordance with accepted academic practice. No use, distribution or reproduction is permitted which does not comply with these terms. 